ARTICLE

\title{
Metal chloride perovskite thin film based interfacial layer for shielding lithium metal from liquid electrolyte
}

\author{
Yi-Chen Yin 1,2,7, Qian Wang 3,7, Jing-Tian Yang ${ }^{4,7}$, Feng Li ${ }^{1}$, Guozhen Zhang (1) 1,3凶 ${ }^{\text {, Chen-Hui Jiang }}{ }^{5}$, \\ Hong-Sheng $\mathrm{Mo}^{2}$, Ji-Song $\mathrm{Yao}^{2}$, Kun-Hua Wang ${ }^{2}$, Fei Zhou (iD ${ }^{1}$, Huan-Xin Ju (id ${ }^{6 凶}$ \& Hong-Bin Yao ${ }^{1,2 凶}$
}

Fabricating a robust interfacial layer on the lithium metal anode to isolate it from liquid electrolyte is vital to restrain the rapid degradation of a lithium metal battery. Here, we report that the solution-processed metal chloride perovskite thin film can be coated onto the lithium metal surface as a robust interfacial layer to shield the lithium metal from liquid electrolyte. Via phase analysis and density functional theory calculations, we demonstrate that the perovskite layer can allow fast lithium ion shuttle under a low energy barrier of $0.45 \mathrm{eV}$ without the collapse of framework. Such perovskite modification can realize stable cycling of $\mathrm{LiCoO}_{2} \mid \mathrm{Li}$ cells with an areal capacity of $2.8 \mathrm{mAh} \mathrm{cm}{ }^{-2}$ using thin lithium metal foil (50 $\left.\mu \mathrm{m}\right)$ and limited electrolyte $\left(20 \mu \mathrm{mAh}^{-1}\right)$ for over 100 cycles at $0.5 \mathrm{C}$. The metal chloride perovskite protection strategy could open a promising avenue for advanced lithium metal batteries.

\footnotetext{
${ }^{1}$ Hefei National Laboratory for Physical Sciences at the Microscale, University of Science and Technology of China, 230026 Hefei, Anhui, China. ${ }^{2}$ Department of Applied Chemistry, University of Science and Technology of China, 230026 Hefei, Anhui, China. ${ }^{3}$ Department of Chemical Physics, University of Science and Technology of China, 230026 Hefei, Anhui, China. ${ }^{4}$ School of the Gifted Young, University of Science and Technology of China, 230026 Hefei, Anhui, China. ${ }^{5}$ CAS Key Laboratory of Materials for Energy Conversion, Department of Materials Science and Engineering, University of Science and Technology of China, 230026 Hefei, Anhui, China. ${ }^{6}$ PHI China Analytical Laboratory, CoreTech Integrated Limited, 402 Yinfu Road, 211102 Nanjing, China. ${ }^{8}$ These authors contributed equally: Yi-Chen Yin, Qian Wang, Jing-Tian Yang. ${ }^{凶}$ email: guozhen@ustc.edu.cn; huanxin.ju@coretechint.com; yhb@ustc.edu.cn
} 
apidly developing electronic devices provide us with better product experience, but simultaneously set a higher standard for energy storage systems ${ }^{1}$. It stimulates extensive search of new materials to meet the increasing demands of highenergy densities of rechargeable batteries ${ }^{2-4}$. With 10 times the specific capacity of graphite ( 3860 versus $370 \mathrm{mAh} \mathrm{g}^{-1}$ ), metallic lithium $(\mathrm{Li})$ is among the most promising candidates for future anode to support high-energy density batteries ${ }^{5,6}$. However, the practical application of $\mathrm{Li}$ metal batteries is hindered by a major issue associated with loose structure of deposited Li during cycling, which would result in accelerated electrolyte "dry-out" failure and probably short circuit of the battery ${ }^{5,7}$.

The solid electrolyte interphase (SEI) layer on the surface of $\mathrm{Li}$ metal anode plays a predominate role in controlling the deposition of $\mathrm{Li}$ metal ${ }^{8,9}$. In the early stage to reinforce the SEI layer, various electrolyte additives, including vinylene carbonate ${ }^{10}$, $\mathrm{LiNO}_{3}{ }^{11}$, and fluorinated ethylene carbonate ${ }^{12}$, have been tried, but the obtained mechanical strength is not enough to suppress the growth of Li dendrites. Lately, the fabrication of artificial SEI protection layer via pre-treatment methodology has been proposed. A series of artificial SEI protection layers, including carbon spheres $^{13}$, polymers ${ }^{14,15}$, alloy layer ${ }^{16}$, composite films ${ }^{17,18}$ have been applied on the surface of Li metal anodes. Despite these artificial SEI layers exhibited the capability to suppress the growth of $\mathrm{Li}$ dendrites ${ }^{16,19}$, the ultra-dense deposition of $\mathrm{Li}$ along with the suppressed detrimental side reactions with liquid electrolyte have not been achieved due to the permeation of liquid electrolyte throughout the protection layers.

Directly fabricating a highly ionic conductive dense thin film on the surface of Li metal anode is a promising route to fully shield the Li metal from liquid electrolyte ${ }^{7,20}$. To design a dense interfacial layer for $\mathrm{Li}^{+}$ion conduction, the framework structure of material should own continuous channels for $\mathrm{Li}^{+}$ion migration ${ }^{21,22}$. The perovskite structure with the general formula $\mathrm{ABO}_{3}$ (where $\mathrm{A}$ is a cation and $\mathrm{B}$ is a divalent metal ion) provides a highly symmetric host to generate a classical $\mathrm{Li}^{+}$ion conductor, lithium lanthanum titanate (LLTO) ${ }^{23}$, but the fabrication of this perovskite host needs high temperature sintering ${ }^{24,25}$, which hindered the application of LLTO thin film on the surface of Li metal anode. Similar to the LLTO perovskite solid electrolyte, other ceramic $\mathrm{Li}^{+}$ion conductors with high shear modulus are also very challenging to be coated as thin films on the Li metal anodes due to their critical preparation conditions ${ }^{26,27}$.

As a new kind of perovskite, metal halide perovskite with a general formula $\mathrm{ABX}_{3}$ ( $\mathrm{X}$ is a halide) can be produced as the form of thin film via a facile solution-processable $\operatorname{method}^{28-30}$. The metal halide perovskites have shown high photoelectric conversion efficiency in planar solar cells and attracted intensive attention in the past decade ${ }^{31}$. Aside from their unique photoelectronic properties, the framework feature of metal halide perovskite can realize the $\mathrm{Li}^{+}$ion intercalation ${ }^{32,33}$, indicating its potential as a fast $\mathrm{Li}^{+}$ion transport material. In addition, to be an electronic insulating layer, metal halide perovskites can get its bandgaps enlarged by using chloride ions as the halide sources ${ }^{34}$. These features of metal chloride perovskite make it as an attractive interfacial material with high $\mathrm{Li}^{+}$ion transport capability to isolate the Li metal anode from liquid electrolyte.

Herein, we develop a solid-state transfer process to apply solution processed metal chloride perovskite thin film as a new type of interfacial layer onto the $\mathrm{Li}$ metal anode. The metal chloride perovskite interfacial layer can allow fast $\mathrm{Li}^{+}$ion transport throughout its framework with a low energy barrier, endowing homogenous ion flux with excellent isolation from liquid electrolyte. Thus, the ultradense deposition of $\mathrm{Li}$ with high areal capacity $\left(30 \mathrm{mAh} \mathrm{cm}^{-2}\right)$ induced by a high current density of $5 \mathrm{~mA} \mathrm{~cm}^{-2}$ is demonstrated and the Li metal anode under the protection of metal chloride perovskite exhibits a stable $\mathrm{Li}$ stripping/plating voltage curve $(<40 \mathrm{mV})$ at the current density of $1 \mathrm{~mA} \mathrm{~cm}^{-2}$ for over $800 \mathrm{~h}$. Attractively, with lean electrolyte $\left(20 \mu \mathrm{mAh}^{-1}\right)$ and thin Li foil $(50 \mu \mathrm{m})$, the $\mathrm{LiCoO}_{2}$ - Li cell with an areal capacity of $2.8 \mathrm{mAh} \mathrm{cm}^{-2}$ can be cycled for more than 100 cycles.

\section{Results}

Shielding mechanism of metal chloride perovskite. Based on the intrinsic feature of metal chloride perovskite, we propose a fast $\mathrm{Li}^{+}$ion transport gradient layer model to illustrate the shielding mechanism of perovskite thin film for the dense deposition of $\mathrm{Li}$ metal (Fig. 1a). At first, the perovskite framework can only allow the entrance of $\mathrm{Li}^{+}$ions, leaving the solvent molecules out, when a negative electric field is applied, because the size of perovskite cavity can only accommodate desolvated $\mathrm{Li}^{+}$ions. After intercalation into the framework, the $\mathrm{Li}^{+}$ions can easily migrate along the contentious vertical channels due to the high symmetry of the perovskite framework. Once the $\mathrm{Li}^{+}$ions approach the interface of perovskite layer and the substrate, a conversion-type electrochemical reaction will occur to form the $\mathrm{Li}-\mathrm{M}$ alloy layer and the insulated $\mathrm{LiCl}$ layer. The $\mathrm{Li}-\mathrm{M}$ layer will promote a homogeneous consequent deposition of $\mathrm{Li}$, which has been confirmed in previous reports ${ }^{16,19}$. In addition, the generated $\mathrm{LiCl}$ can insulate electrons and prevent the perovskite from further conversion reaction, retaining the stability of the perovskite on the top surface. Therefore, the finally formed solid electrolyte interfacial layer is a gradient thin film consisting of perovskite framework on the top surface to isolate the liquid electrolyte and underneath $\mathrm{Li}-\mathrm{M}$ alloy layer to induce the homogeneous deposition of $\mathrm{Li}$ metal.

To unravel the conduction mechanism of $\mathrm{Li}^{+}$ion in the perovskite lattice, we performed density functional theory (DFT) calculation to simulate the migration of $\mathrm{Li}^{+}$ions in the perovskite framework (Supplementary Note 1), obtaining the specific migration pathways and associated energy barriers (Supplementary Fig. 1). The simulated model is based on the cubic phase $\mathrm{MASnCl}_{3}$ ( $\mathrm{MA}=$ methylammonium, $\mathrm{CH}_{3} \mathrm{NH}_{3}$ in formula) and the potential energy surface along the migration pathway of $\mathrm{Li}^{+}$ ion is shown in Fig. 1b. The corresponding positions of $\mathrm{Li}^{+}$ion at its initial state, transition state and final state are displayed as the insets. To accommodate the migration of $\mathrm{Li}^{+}$ions, the lattice undergoes mild yet measurable distortion, accompanied by the orientation adjustments of MA ion due to the nonuniform distribution of electric potential in the lattice (Supplementary Fig. 2). During the diffusion process, the transition state at the peak of potential energy curve is reached as the $\mathrm{Li}^{+}$ion is about to pass through the shared face of two neighboring unit cells of perovskite. Interestingly, the MA ion continues adjusting its orientation along with the migration of $\mathrm{Li}^{+}$ion and restores its original direction as $\mathrm{Li}^{+}$ion enters another unit cell. This is crucial for $\mathrm{Li}^{+}$ion to maintaining a repeatable migration in the perovskite lattice and enabling the $\mathrm{Li}^{+}$ion conduction throughout the perovskite framework. The estimated energy barrier is $0.45 \mathrm{eV}$, close to the activation energy barrier of a couple of other known solid-state-electrolytes (e.g., $0.53 \mathrm{eV}$ for $\mathrm{Li}_{4} \mathrm{GeS}_{4}, 0.49 \mathrm{eV}$ for $\gamma-\mathrm{Li}_{3} \mathrm{PS}_{4}$, to name a few $)^{35}$, indicating the ease of $\mathrm{Li}^{+}$ion conduction in the metal chloride perovskite framework.

To demonstrate the gradient layer model of the perovskite, we fabricated the metal chloride perovskite thin films composed of $\mathrm{MASnCl}_{3}$ or $\mathrm{MAPbCl}_{3}$ with high film quality on the substrates via solution spin-coating technique at first (see Methods and Supplementary Fig. 3). The thickness of as-fabricated perovskite film is $\sim 1 \mu \mathrm{m}$ for $\mathrm{MASnCl}_{3}$ and $\sim 2 \mu \mathrm{m}$ for $\mathrm{MAPbCl}_{3}$ (Supplementary Fig. 4). The X-ray diffraction (XRD) patterns (Supplementary Fig. 5) show that the pure perovskite thin films possess 

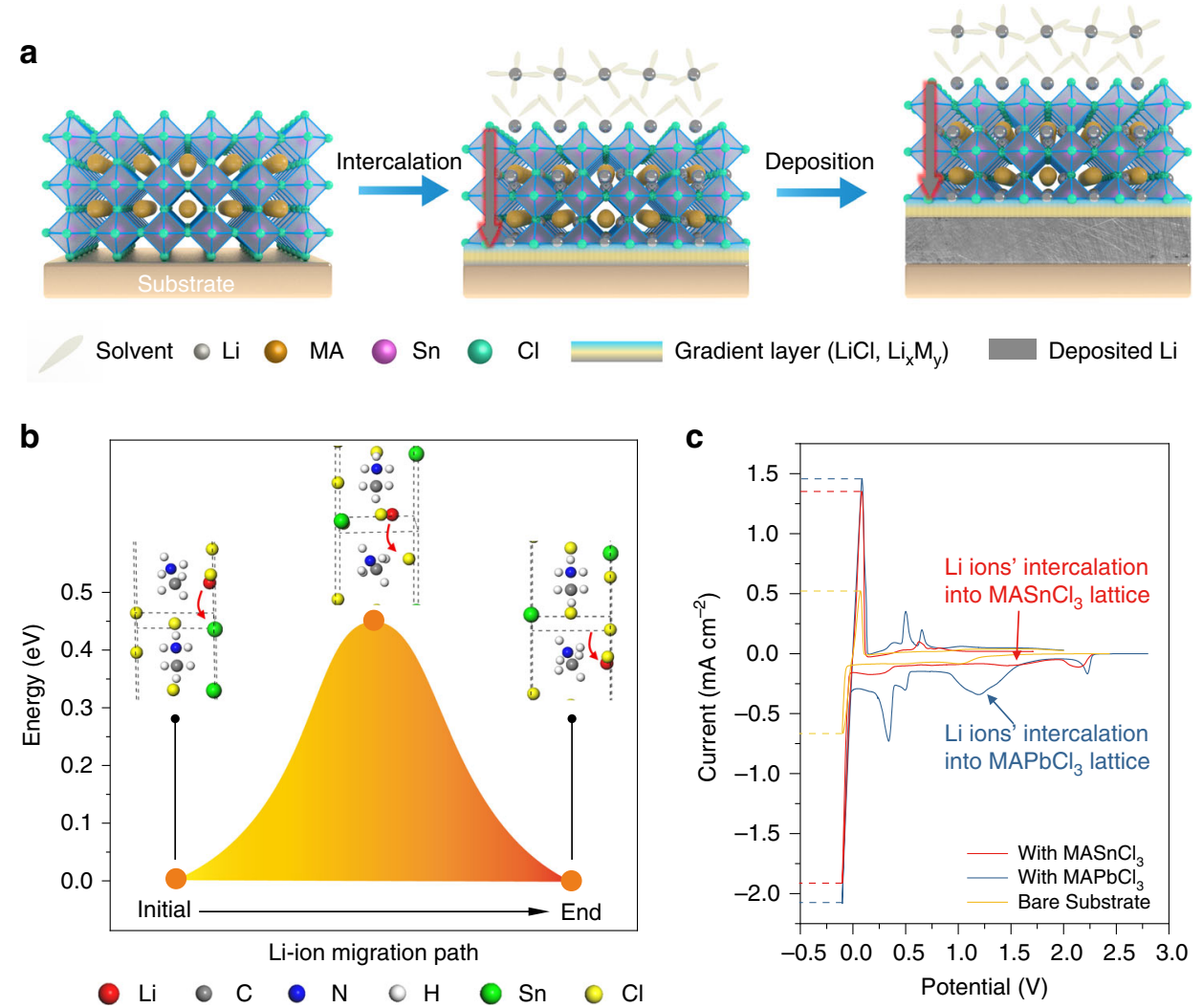

Fig. 1 Mechanism exploration of $\mathrm{Li}^{+}$ion migration through the lattice of metal chloride perovskite for shielding. a Schematic illustration of the mechanism of Li ions' intercalation into perovskite lattice, the formation of perovskite-alloy gradient Li ion conductor and the deposition process. $\mathbf{b}$ hypothetical migration pathway of $\mathrm{Li}^{+}$ion and corresponding potential energy surface highlighting the initial state, peak state and final state in a period. $\mathbf{c}$, Cyclic voltammetry comparison of the cells using $\mathrm{MASnCl}_{3}$-coated, $\mathrm{MAPbCl}_{3}$-coated or bare substrate as the working electrode, and the lithium as the counter and reference electrode with the sweep rate of $1 \mathrm{mV} \mathrm{s}^{-1}$.

highly oriented crystalline structure with a series of diffraction peaks belonging to crystal indices of $(\mathrm{n} 00)(n=1,2,3 \ldots)$. Such highly oriented crystalline structure is consistent with our proposed framework structure in Fig. 1a, which will facilitate the $\mathrm{Li}^{+}$ion transport throughout the perovskite layer.

We investigated the cyclic voltammetry (CV) of Li plating on these thin film-coated substrates and compared them to that of bare substrate. As shown in Fig. 1c, the perovskite film coated substrates experience an intercalation of $\mathrm{Li}^{+}$ions appearing at the potential peak higher than $1 \mathrm{~V}\left(\sim 1.5 \mathrm{~V}\right.$ for $\mathrm{MASnCl}_{3}$, and $\sim 1.2 \mathrm{~V}$ for $\mathrm{MAPbCl}_{3}$ ) in comparison to the flat current curve in the CV of bare substrate without the characteristic $\mathrm{Li}^{+}$ion intercalation process. The other CV peaks with the potential around $0.4-0.7 \mathrm{~V}$ belong to the reduction process of interfacial perovskite to $\mathrm{Li}-\mathrm{Sn}$ or Li-Pb alloy, and the final state of as-formed alloy is $\mathrm{Li}_{17} \mathrm{Sn}_{4}$ or $\mathrm{Li}_{17} \mathrm{~Pb}_{4}{ }^{36-38}$. As the potential approaches to $-0.1 \mathrm{~V}$, the current density of the substrate with $\mathrm{MASnCl}_{3}$ or $\mathrm{MAPbCl}_{3}$ is around 1.5 $\mathrm{mA} \mathrm{cm}{ }^{-2}$, much higher than the current density of bare substrate $\left(\sim 0.6 \mathrm{~mA} \mathrm{~cm}^{-2}\right)$. Similarly, at the symmetric voltage position near $+0.1 \mathrm{~V}$, the perovskite-modified substrates also present much higher response currents, indicating the promotion of $\mathrm{Li}$ plating/stripping kinetics by the perovskite thin films. To further show the unique role played by the perovskite framework rather than as-formed $\mathrm{Li}-\mathrm{M}$ alloy layer, we compared the $\mathrm{CV}$ curves of $\mathrm{MAPbCl}_{3}$ and $\mathrm{PbCl}_{2}$-coated substrates (Supplementary Fig. 6). The absence of typical $\mathrm{Li}^{+}$ion intercalation peak around $1.2 \mathrm{~V}$ and much lower Li platting/stripping current density of $\mathrm{PbCl}_{2}-$ coated substrate in comparison to that of $\mathrm{MAPbCl}_{3}$ coated substrate demonstrated the unique function of perovskite structure to promote Li plating/striping kinetics.

To confirm the self-prevented electrochemical reduction of perovskites, we evaluated the amount of electrochemically reduced perovskites through galvanostatic lithiation tests (Supplementary Fig. 7). The calculated results (Supplementary Note 2) showed that only a small part of perovskites ( $\sim 30 \%$ for $\mathrm{MASnCl}_{3}$ and $15 \%$ for $\mathrm{MAPbCl}_{3}$ ), corresponding to the absolute quantities (about $2.8 \times 10^{-7} \mathrm{~mol} \mathrm{~cm}^{-2}$, or $0.3 \mu \mathrm{m}$ in thickness) of perovskites, was reduced to form $\mathrm{Li}_{17} \mathrm{Sn}_{4}$ or $\mathrm{Li}_{17} \mathrm{~Pb}_{4}$ layer. The limited perovskite consumption independent of the original thickness values of perovskite layer implies that the perovskite thin films are not completely reduced due to the formation of insulating $\mathrm{LiCl}$ layer, as well as the intrinsic property of wide band gap of metal chloride perovskite to isolate electrons coming from the current collector. Therefore, the finally formed gradient interfacial layer is consisted of perovskite framework on the top surface followed by the $\mathrm{Li}-\mathrm{M}$ alloy/LiCl layer beneath, which is evidenced by the depth X-ray photoelectron spectroscopy (XPS) analysis (Supplementary Fig. 8 and Supplementary Note 3).

Ultra-dense $\mathrm{Li}$ deposition underneath metal chloride perovskite. To demonstrate practical protection role of metal chloride perovskite, we investigated the $\mathrm{Li}$ plating behavior underneath these metal chloride perovskite thin films. We directly applied Li plating onto the perovskite-coated substrates in half cells (see Methods and Supplementary Fig. 9a). As shown in Fig. 2a, after 
a

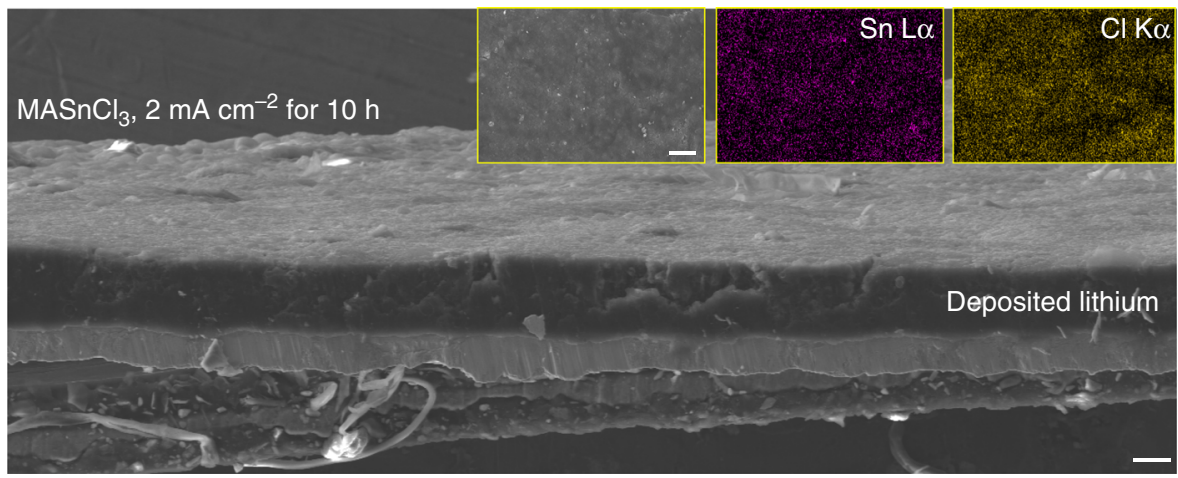

b

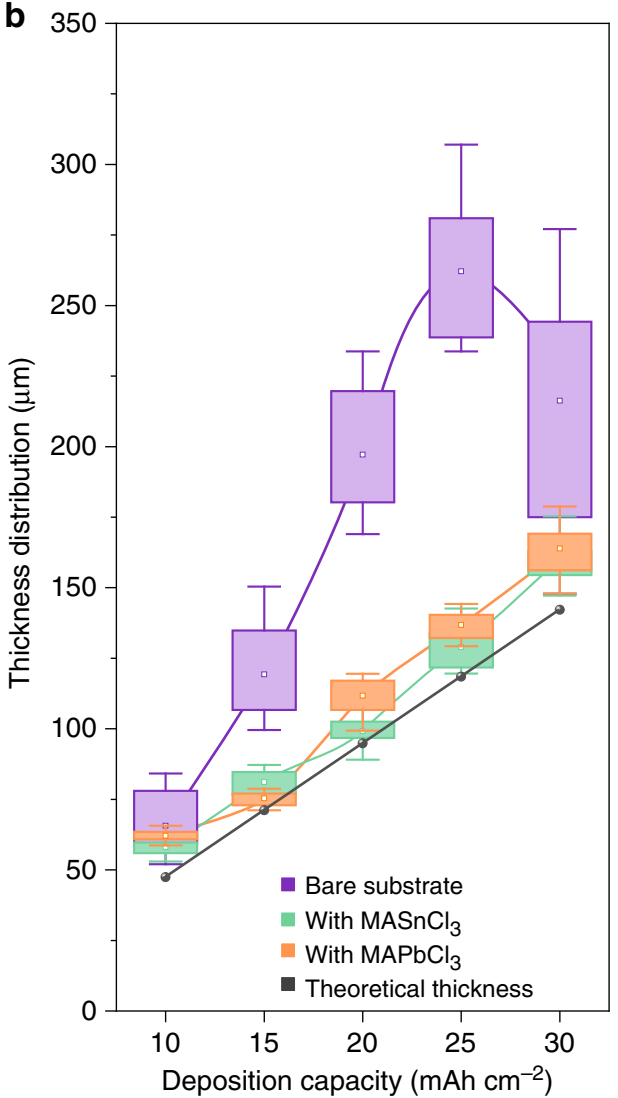

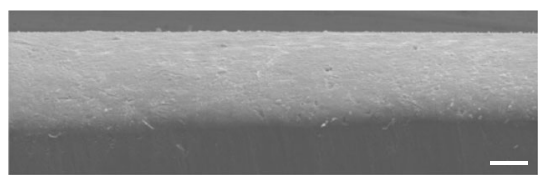

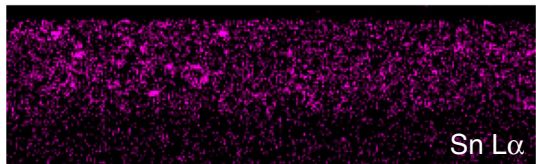

d

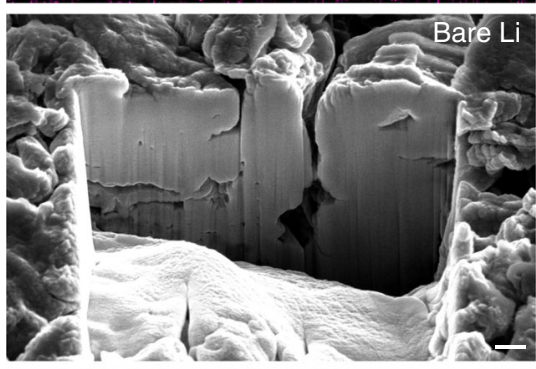

e

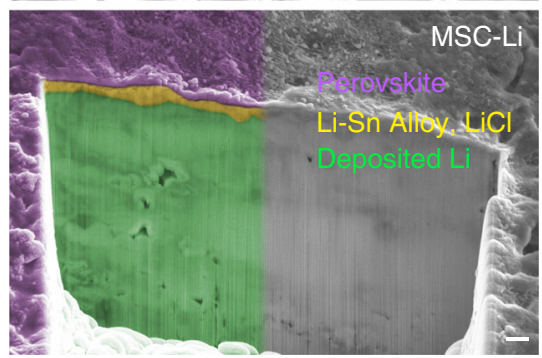

Fig. 2 Ultra-dense Li deposition enabled by high-quality perovskite thin film. a Morphologies of Li electrodeposited on the $\mathrm{MASnCl}_{3}$-coated substrate, and the SEM image of the top surface with corresponding EDX mappings indicating the distribution of $\mathrm{Sn}, \mathrm{Cl}$ on the top surface of as-deposited Li. b Thickness statistics of Li electrodeposited on the bare (purple), $\mathrm{MASnCl}_{3}$-coated (green) or $\mathrm{MAPbCl}_{3}$-coated (orange) substrate. Theoretical thickness of $\mathrm{Li}$ is set as a reference (black). Current densities range from 2 to $6 \mathrm{~mA} \mathrm{~cm}{ }^{-2}$ and the deposition time lasted for $5 \mathrm{~h}$. c Side-view SEM image and corresponding EDX mapping indicating the distribution of Sn on the surface of MSC-Li. d e FIB-processed deep cross-sectional morphologies of bare Li (d) and MSC-Li (e) after electrodeposition under $4 \mathrm{~mA} \mathrm{~cm}^{-2}$ for $2 \mathrm{~h}$, where the different color based on the contrast difference implies the retention of perovskite-alloy-lithium (purple-yellow-green) gradient structure of MSC-Li. Scale bars: $50 \mu \mathrm{m}$ in a, $100 \mu \mathrm{m}$ in $\mathrm{c}$, and $1 \mu \mathrm{m}$ in $\mathbf{d}$, e.

the Li plating under $2 \mathrm{~mA} \mathrm{~cm}^{-2}$ for $10 \mathrm{~h}$, an ultra-dense and dendrite-free $\mathrm{Li}$ metal deposition is observed underneath the $\mathrm{MASnCl}_{3}$ perovskite thin film. The energy dispersive X-ray spectroscopy (EDX) analyses (Insets in Fig. 2a) confirmed that the top surface of the substrate after the Li plating is still composed of $\mathrm{Sn}$ and $\mathrm{Cl}$, which indicates that the $\mathrm{Li}$ plating was processed underneath the perovskite thin film. In order to confirm that the perovskite film was not completely reduced during electrochemical Li deposition, XPS was performed to examine the chemical state of Sn (Supplementary Fig. 9b). After Li deposition, the $\mathrm{Sn} 3 \mathrm{~d}$ spectrum of $\mathrm{MASnCl}_{3}$-coated substrate shows higher binding energy peaks of oxidation state of Sn than that of zero valence state $\mathrm{Sn}^{0}$, indicating no reduction of $\mathrm{MASnCl}_{3}$ on the top surface. Moreover, XRD pattern of the deposited $\mathrm{Li}$ on $\mathrm{MASnCl}_{3}$ coated substrate confirmed the obtained lithiated perovskite with highly oriented crystal structure (Supplementary Fig. 10b), implying that the formed lithiated perovskite thin film inherited the highly oriented crystal structure from original $\mathrm{MASnCl}_{3}$ thin film. The similar dense Li deposition behavior can be also achieved underneath the $\mathrm{MAPbCl}_{3}$ perovskite thin film with retained high crystal orientation (Supplementary Figs. 9c, 10c, and 11).

We further carried out the Li plating on $\mathrm{MASnCl}_{3}$ or $\mathrm{MAPbCl}_{3}$ coated substrates under the current densities from $2 \mathrm{~mA} \mathrm{~cm}^{-2}$ to $6 \mathrm{~mA} \mathrm{~cm}^{-2}$ for $5 \mathrm{~h}$. The dense structure of plated Li metal can be observed underneath all metal chloride perovskite layers even under the current density up to $6 \mathrm{~mA} \mathrm{~cm}^{-2}$ (Supplementary 
Fig. 12). The top surfaces of all perovskite-modified Li deposits exhibit smooth appearance without any dendrites (Supplementary Fig. 13). In contrast, the Li plated on the bare substrate presents a highly porous structure and obvious dendrites. We measured the thickness of the plated Li and correlated it with the areal capacity of $\mathrm{Li}$ as plated (Supplementary Tables 1 and 2). As shown in Fig. 2b, the thickness of Li deposited underneath metal chloride perovskite films shows nearly the same linear relationship with the areal capacity as that of theoretical value of $100 \%$ dense Li (Supplementary Note 4). In contrast, the Li plated on the bare substrate exhibits exponential increase of thickness with the area capacity. The abnormal decline of the thickness of $\mathrm{Li}$ deposited on the bare substrate at $6 \mathrm{~mA} \mathrm{~cm}^{-2}$ can be attributed to the serious dendrite growth and pulverization causing the loss of "dead Li" in the electrolyte.

To apply the perovskite thin film onto practical Li metal anode, we developed a solid-state pressing process to transfer perovskite thin film from the substrate onto the surface of $\mathrm{Li}$ metal (see Methods and Supplementary Fig. 14). As-transferred perovskite thin film can build up an intimate contact with $\mathrm{Li}$ metal via the in situ formation of gradient perovskite/Li-M alloy layer (Supplementary Note $5, \mathrm{M}=\mathrm{Sn}$ or $\mathrm{Pb}$ ), accompanied by spontaneous lithiation of perovskite (Supplementary Fig. 15 and Supplementary Note 6). The unique gradient structure of the asfabricated perovskite/Li-M alloy layer was evidenced by depth analysis of XPS (Supplementary Figs. 16, 17, and 18 and Supplementary Note 7). The solid-state transfer procedure is well compatible with traditional electrode processing, which has the potential to be applied in large scale (Supplementary Fig. 19). The fabricated $\mathrm{MASnCl}_{3}$-coated $\mathrm{Li}$ metal anode and $\mathrm{MAPbCl}_{3}$-coated $\mathrm{Li}$ metal anode are here denoted as MSC-Li and MPC-Li, respectively. As shown in Fig. 2c and Supplementary Fig. 20, the $\mathrm{MASnCl}_{3}$ film and $\mathrm{MAPbCl}_{3}$ film are tightly and uniformly coated on the surface of Li metal anodes.

Focused ion beam (FIB) was performed to deeply expose the cross-sectional morphologies of bare $\mathrm{Li}$ and MSC-Li after $2 \mathrm{~h} \mathrm{Li}$ plating under a current density of $4 \mathrm{~mA} \mathrm{~cm}^{-2}$. Dense structure of $\mathrm{Li}$ deposit is observed in the MSC-Li anode in comparison to the porous structure of that of bare $\mathrm{Li}$ metal anode (Fig. 2d, e), implying that the transferred $\mathrm{MASnCl}_{3}$ layer can well induce ultra-dense Li deposition. Notably, MSC-Li exhibits an obvious contrast difference (denoted by purple, yellow and green) in vertical direction near the surface, implying a perovskite-alloylithium interfacial layer on the Li metal anode surface to resist the liquid electrolyte corrosion. Similarly, the ultra-dense Li deposition can be also realized on the MPC-Li anode with the retention of perovskite protection layer (Supplementary Fig. 21). In addition, optically transparent cell tests (experiment details are shown in Supplementary Fig. 22 and Methods) also indicated that the Li dendrite growth can be well suppressed by the perovskitemodified layer in comparison to unmodified $\mathrm{Li}$ metal anode (Supplementary Fig. 23).

Stability of metal chloride perovskite coated Li metal anode. To evaluate the stability of perovskite coated $\mathrm{Li}$ metal anode, we carried out the galvanostatic $\mathrm{Li}$ plating/stripping test on the symmetric cell. The impedance spectra of symmetric cells using different electrodes before cycling are firstly compared. As shown in Fig. 3a, much lower interfacial resistance values of the cells with MSC-Li $(\sim 60 \Omega)$ or MPC-Li $(\sim 85 \Omega)$ are obtained in comparison to that of the cell with bare Li electrode $(\sim 320 \Omega)$, indicating the stability of perovskite interlayer to promote the charge transfer at the electrode interface. In the following cycling test (Fig. 3b), the MSC-Li cell can be cycled for more than $800 \mathrm{~h}$, much longer than the $100 \mathrm{~h}$ cycling life of the cell using bare $\mathrm{Li}$.
The detailed voltage profiles show that the stable overpotential with a low value of $40 \mathrm{mV}$ can be maintained in the cell using MSC-Li electrode. The bare Li electrode, however, exhibits a drastic voltage increase at $100 \mathrm{~h}$, which can be attributed to poor $\mathrm{Li}^{+}$ion transfer kinetics caused by as formed nonuniform SEI layer. The symmetric cell of MPC-Li electrodes also shows attractive advantages with low overpotential of around $40 \mathrm{mV}$ and a stable cycling life of over $600 \mathrm{~h}$ (Supplementary Fig. 24).

Furthermore, we applied XRD analysis to validate the framework stability of highly oriented perovskite layer on the Li metal anode during the cycling. As shown in Fig. 3c, before cycling, the XRD pattern of MSC-Li (blue curve) displayed a series of peaks with indices of (100) and (200) planes of the lithiated perovskite. The other peaks in the XRD pattern can be assigned to $\mathrm{Li}$ (marked with "o"). More attractively, after 100 cycles, the MSC-Li still retains a fine correspondence of the XRD pattern (red curve) as that before cycling, demonstrating that the perovskite framework can maintain its structural orientation and behave good stability against the electrolyte corrosion under the electrochemical operation. Similarly, the perovskite framework on the MPC-Li electrode can also keep the phase stability during the electrochemical cycling (Supplementary Fig. 25), further showing the robustness of metal chloride perovskite as the solid interphase layer. By comparing to raw spincoated perovskite, the as-fabricated lithiated perovskites exhibit no obvious XRD peak shift (Supplementary Fig. 26), implying the good capability of perovskite framework to accommodate $\mathrm{Li}^{+}$ions. The phenomenon is in good agreement with previous works in which the doping and intercalation of $\mathrm{Li}^{+}$ions into perovskite framework did not cause significant change in crystal unit cell size 39,40 (Supplementary Fig. 27).

To further explore chemical stability of perovskite layer on the Li metal anode, we conducted XPS analysis on the top surface of MSC-Li electrode before and after cycling. The Sn $3 d$ XPS spectra of MSC-Li are shown in Fig. 3d, where the reference metallic Sn $3 d_{5 / 2}$ is marked by dotted line at the binding energy of $484.8 \mathrm{eV}$. Before cycling, the $S n 3 d_{5 / 2}$ peak is found to be at $486.6 \mathrm{eV}$, which belongs to the oxidation state of Sn, obviously different from that of metallic Sn. After 100 cycles, it is obvious that the XPS peak of Sn $3 d$ stayed at the same position $\left(486.6 \mathrm{eV}\right.$ for $\left.\mathrm{Sn} 3 \mathrm{~d}_{5 / 2}\right)$, which indicates the chemical stability of perovskite on the top surface of MSC-Li and high consistence with the XRD result. The XPS result is similar for MPC-Li electrode, showing that the lead on the top surface of MPC-Li electrode always retained the oxidation state before and after cycling (Supplementary Fig. 28). All these results indicate that the perovskite layer on the top surface of the $\mathrm{Li}$ metal was not reduced to the alloy layer during the cell cycling.

In addition, the stabilities of the perovskite modified electrodes are revealed by the surface morphologies analysis after $100 \mathrm{~h}$ cycling. The MSC-Li electrode displays highly dense surface morphology with silvery shining appearance after cycling, in contrast to the drastic roughness and black surface of bare Li electrode (Supplementary Fig. 29). The EDX mapping images of oxygen distribution on the surface of the MPC-Li electrode before and after cycling further reveal that the perovskite film is still dense to endow Li metal with the resistance against oxidation under the exposure to air during the sample transfer for SEM characterization (Supplementary Fig. 30). The EIS curves of the above symmetric cells after cycling in Supplementary Fig. 31 also confirm the advantage of the perovskite modification to facilitate $\mathrm{Li}^{+}$ion transport.

Performance of Li metal battery protected by metal chloride perovskite. To demonstrate the efficiency of perovskite protection for $\mathrm{Li}$ metal batteries, we tested the electrochemical performance of $\mathrm{Li}_{4} \mathrm{Ti}_{5} \mathrm{O}_{12}$ (LTO)/perovskite coated Li cells at a high rate 


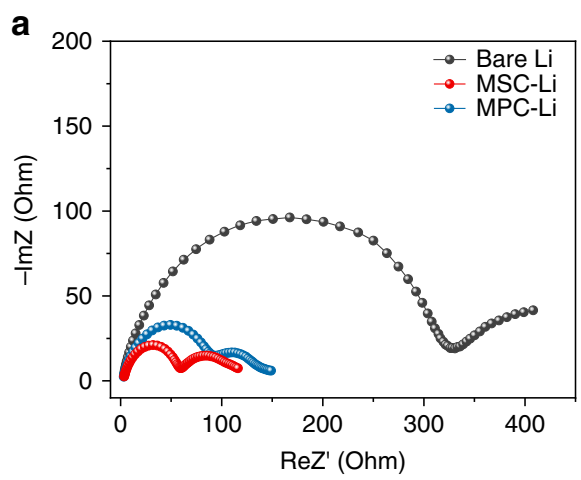

C

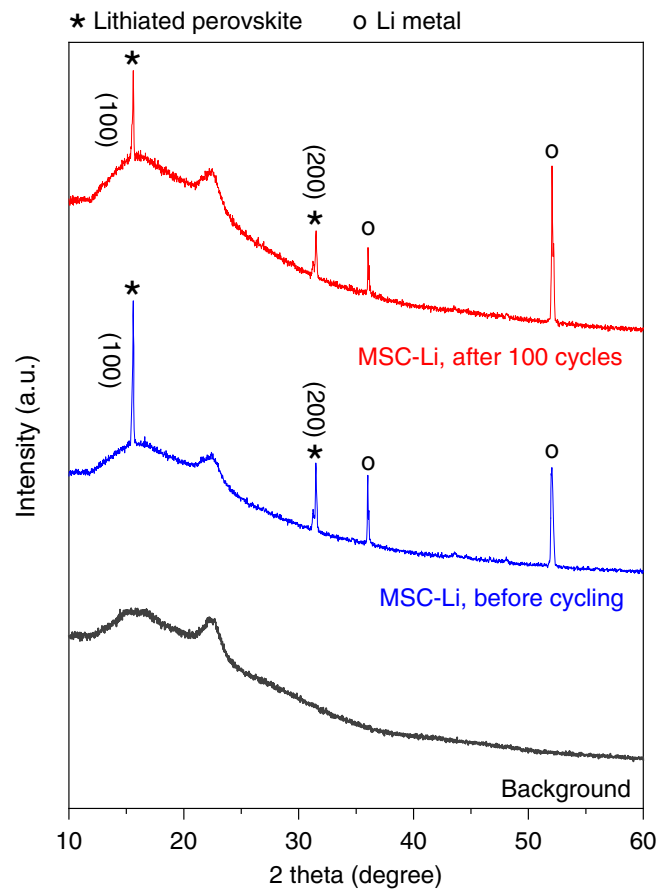

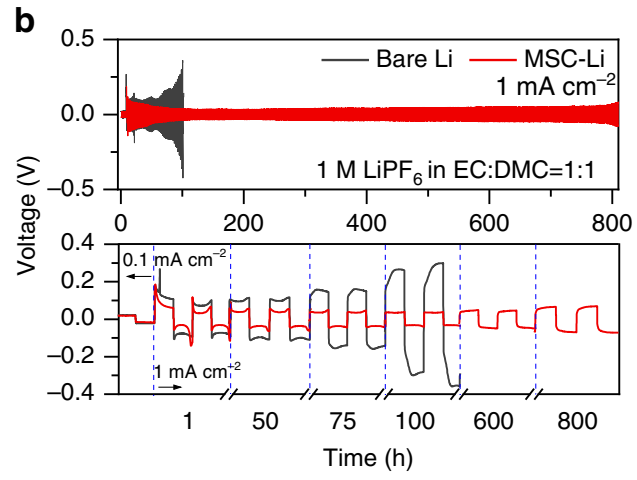

d

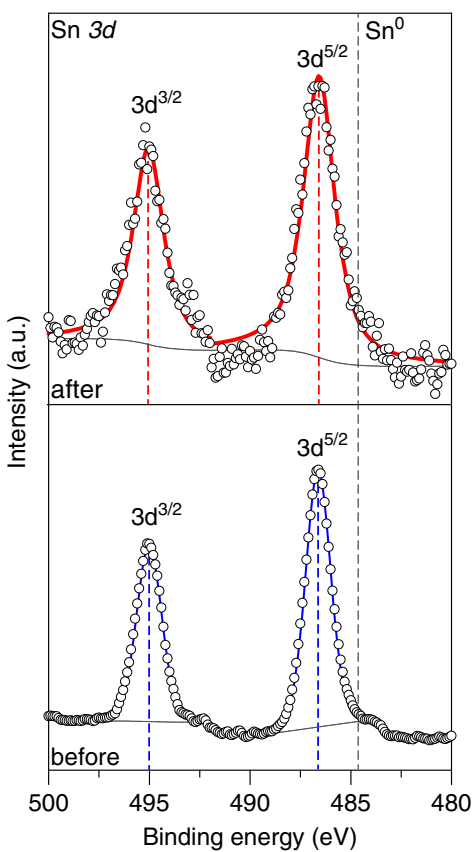

Fig. 3 Electrochemical stability of MSC-Li and MPC-Li. a EIS spectra of bare Li, MSC-Li and MPC-Li. b Galvanostatic voltage curves (top) of bare Li and MSC-Li tested with a current density of $1 \mathrm{~mA} \mathrm{~cm}^{-2}$ for $1 \mathrm{mAh} \mathrm{cm} \mathrm{cm}^{-2}$, and the enlarged voltage curves during different periods (bottom). c XRD patterns of MSC-Li anode before cycling (blue) and after 100 cycles (red) to indicate the electrochemical stability and phase retention of lithiated perovskite during cycling, accompanied with the XRD pattern of sealing tape to isolate samples from air (black). d XPS spectra of the surface of MSC-Li anode before cycling (blue) and after 100 cycles (red) to prove that the perovskite solid-electrolyte interface stays on the top surface after cycling.

of 5 C. As shown in Fig. 4a, the cell with bare Li metal anode exhibits a drastic capacity decay and the capacity decreased to $28.3 \mathrm{mAh} \mathrm{g}^{-1}$ at 400 th cycle. In contrast, the cells using MSC-Li and MPC-Li anode show stable cycling for more than 500 cycles with a low capacity decay rate of $0.07 \%$ per cycle. The charge/ discharge voltage profiles in Fig. $4 \mathrm{~b}$, $\mathrm{c}$ show that the charge voltage plateau of the cell using bare Li metal anode is much higher than that of MSC-Li anode, indicating that the perovskite layer promotes the kinetics of Li plating. The discharge voltage plateau of the cell using bare $\mathrm{Li}$ anode becomes lower and lower during cycling in comparison to the stable discharge voltage plateau of the cell with MSC-Li, implying a higher resistance for Li stripping caused by deteriorated surface of bare Li anode. The surface SEM images of $\mathrm{Li}$ anodes were taken at 400th cycle to further show the protection role played by the perovskite thin films (Fig. $4 \mathrm{~d}-\mathrm{f}$ ). Severe pulverization and loose morphology were observed on the surface of bare Li anode, whereas the surface of MSC-Li or MPCLi anode still exhibited typical smooth morphologies, demonstrating the robustness of perovskite protection layer in practical Li metal batteries. The impedance spectra of the three cells before and after 400 cycles are compared in Supplementary Fig. 32, also indicating a stabilized interface of MSC-Li and MPC-Li compared to bare Li metal anode. Additionally, the surface SEM image and corresponding EDS mapping of MPC-Li in Li|| LTO cell after 500 cycles were also taken. As shown in Supplementary Fig. 33, most surface area of MPC-Li is smooth and dense after cycling, revealing good stability of the perovskite layer.

We further explored the performance of perovskite protected Li metal battery by applying strict conditions including high-areal

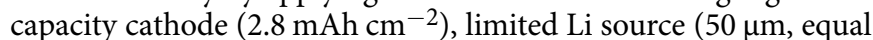
to $10 \mathrm{mAh} \mathrm{cm}^{-2}$ ) and lean electrolyte $\left(20 \mu \mathrm{mAh}^{-1}\right)$ in the $\mathrm{LiCoO}_{2} \mid \mathrm{Li}$ cell at $0.5 \mathrm{C}$. As shown in Fig. $4 \mathrm{~g}$, the cell using bare $\mathrm{Li}$ metal anode exhibits a rapid capacity decay more than $40 \%$ after only 20 cycles. By contrast, MSC-Li and MPC-Li anodes enable the cells to maintain a stable capacity performance for over 100 cycles with a capacity retention of $85 \%$. It indicates that perovskite protective layers can effectively isolate the Li metal from the liquid electrolyte to restrain Li loss and side reactions. In addition, a much lower polarization effect was also observed in the discharge/charge voltage profile of the cell using perovskite 


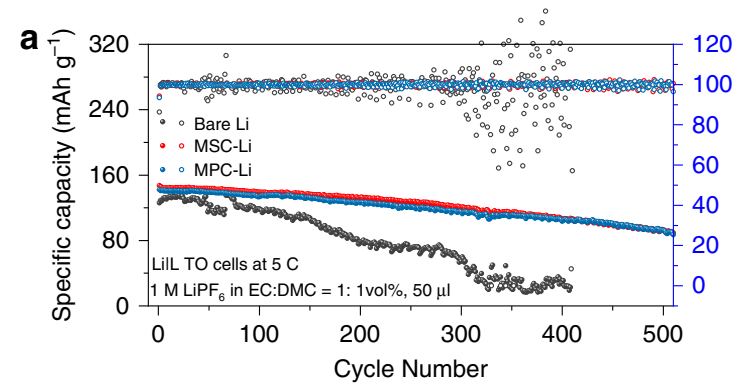

b
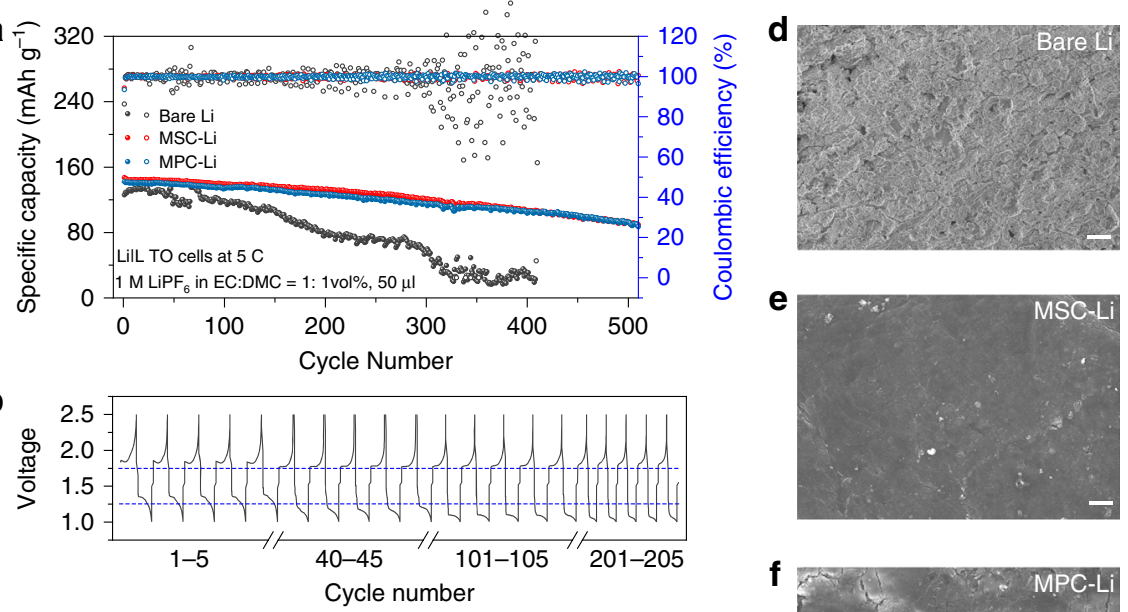

C
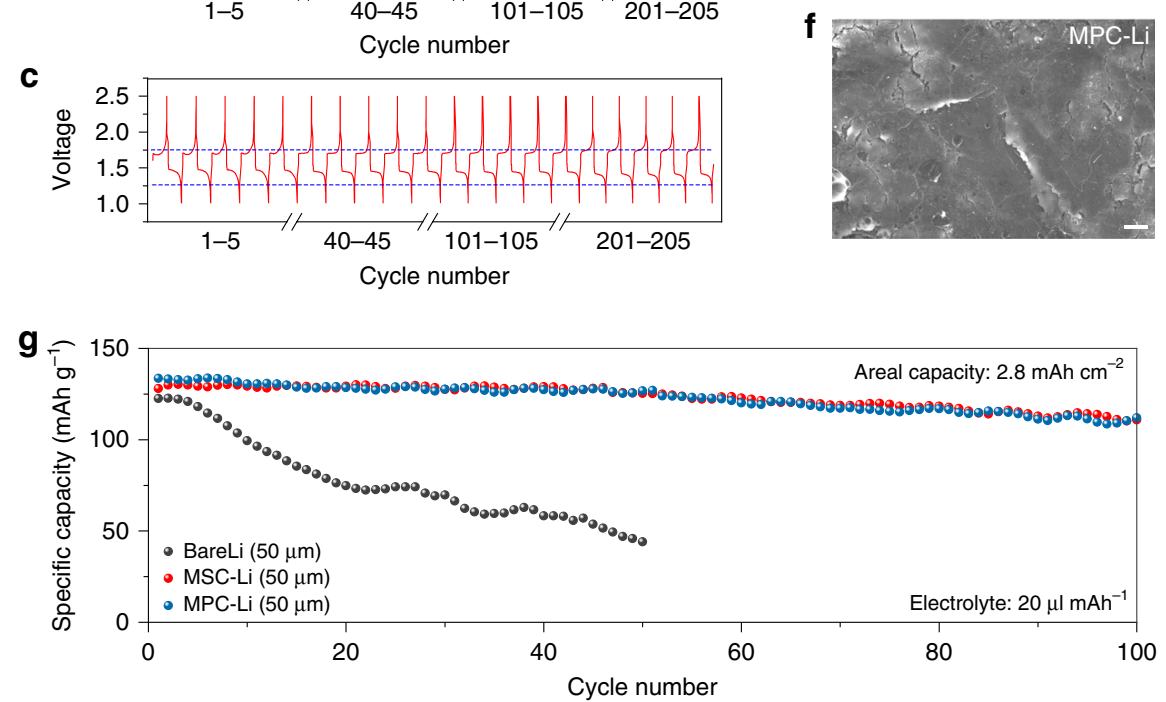

Fig. 4 Electrochemical performances of Li|LTO cells at $\mathbf{5} \mathbf{C}$ and Li|LCO cells at $\mathbf{0 . 5} \mathbf{C}$. a Galvanostatic cycling performances of cells using $\mathrm{Li}_{4} \mathrm{Ti}_{5} \mathrm{O}_{12}$ as cathode and bare Li, MSC-Li or MPC-Li as anode. b, c Voltage curves of bare Li (b) and MSC-Li (c) at different cycles. d-f, Surface morphologies of bare Li (d), MSC-Li (e), and MPC-Li (f) anodes after 400 cycles. $\mathbf{g}$ Galvanostatic cycling performances of cells using $\mathrm{LiCoO}_{2}$ as cathode and $50 \mu \mathrm{m}$ thick bare Li, MSC-Li or MPC-Li as anode. Scale bars: $50 \mu \mathrm{m}$.

modified Li anode (Supplementary Fig. 34), further demonstrating the good protection of the perovskite thin film for the $\mathrm{Li}$ metal anode.

\section{Discussion}

In summary, we have demonstrated that metal chloride perovskite thin films can be used as stable interfacial layers to stabilize the Li metal anode. Due to the electrochemical stability and highly oriented framework for fast $\mathrm{Li}^{+}$ion conduction, the metal chloride perovskite thin film can not only act as efficient solid electrolyte interlayer to induce ultra-dense deposition of $\mathrm{Li}$ underneath their protection, but also isolate the Li metal anode from liquid electrolyte to provide a favorable environment for stable Li plating/stripping, effectively restraining the loss of lithium and the electrolyte during cycling. Furthermore, the metal chloride perovskite protection layer can enable stable cycling of $\mathrm{Li}$ metal battery under strict conditions. This innovative strategy to apply solution-processed metal chloride perovskite thin films as interfacial layers on the $\mathrm{Li}$ metal anodes will inspire more exploration of metal halide perovskites as ion transport materials, pushing ahead the development of advanced energy storage systems.

\section{Methods}

Fabrication of metal chloride perovskite thin films. Stainless steel foil (50 or $100 \mu \mathrm{m}$ ) was washed with deionized water, acetone and ethanol in an ultrasonic cleaner (SCIENTZ, SB25-12DTD, 40k HZ) sequentially before drying in an oven at $70{ }^{\circ} \mathrm{C}$ for $30 \mathrm{~min}$. For $\mathrm{MASnCl}_{3}$ perovskite thin films, $0.338 \mathrm{~g}$ of methylamine hydrochloride ( $\mathrm{MACl}, 98.5 \%$, Macklin) and $0.948 \mathrm{~g}$ of stannous(II) chloride ( $\mathrm{SnCl}_{2}, 99.9 \%$, Aladdin) were dissolved in $10 \mathrm{~mL}$ of $\mathrm{N}, \mathrm{N}$-dimethylformamide (DMF, 99.9\%, Aladdin) to form $\mathrm{MASnCl}_{3}$ precursor solution with a concentration of $0.5 \mathrm{M}$. $0.676 \mathrm{~g}$ of methylamine hydrochloride (MACl, 98.5\%, Macklin). For $\mathrm{MAPbCl}_{3}$ perovskite thin films, $2.781 \mathrm{~g}$ of Lead chloride $\left(\mathrm{PbCl}_{2}, 99.9 \%\right.$, Aladdin) were dissolved in $10 \mathrm{~mL}$ dimethyl sulfoxide (DMSO, 99.9\%, Aladdin) to form $\mathrm{MAPbCl}_{3}$ solution with a concentration of $1 \mathrm{M}$. Each type of as-prepared perovskite precursor solution was spun-coated onto UV-ozone treated stainless steel foil substrate $\left(50 \mu \mathrm{l}\right.$ for every $1 \mathrm{~cm}^{-2}$ of substrate area) at $2000 \mathrm{r} / \mathrm{min}$ for $90 \mathrm{~s}$. Then the perovskite coated stainless steel foils were annealed at $70^{\circ} \mathrm{C}$ for $5 \mathrm{~min}$. All the $\mathrm{MASnCl}_{3}$ and $\mathrm{MAPbCl}_{3}$ thin films were fabricated and stored in an argon-filled glove box with $<1$ ppm $\mathrm{O}_{2}$ and $<1 \mathrm{ppm} \mathrm{H}_{2} \mathrm{O}$.

Solid-state transfer process to form MSC-Li and MPC-Li anodes. Li metal foil (99.9\%, China Energy Lithium Co., Ltd.) was polished to remove the oxidation layer to obtain a smooth and shinning surface. Then, the as-treated $\mathrm{Li}$ foil was pressed onto the perovskite-coated substrate with a pressure of $10 \mathrm{~N} \mathrm{~cm}^{-2}$ for $10 \mathrm{~s}$. The perovskite thin film can be easily transferred from the stainless steel substrate onto the surface of Li metal after separating the Li foil from the stainless steel substrate. MSC-Li and MPC-Li electrodes are thus obtained. The transfer operations were performed at the room temperature of $25^{\circ} \mathrm{C}$ in the glove box with $<1$ ppm $\mathrm{O}_{2}$ and $<1$ ppm $\mathrm{H}_{2} \mathrm{O}$. 
Material characterizations. XRD measurements were carried out on a XPERTPRO powder X-ray diffractometer operating at $40 \mathrm{kV}$ and $30 \mathrm{~mA}$ with a scanning step of 0.02 degree, using $\mathrm{Cu} \mathrm{K}$ radiation $(\lambda=0.15406 \mathrm{~nm})$. The samples were tested in the Kapton tape-sealed devices for the XRD characterizations (Supplementary Fig. 10a). The micro-morphologies were observed with SU8220 (HITACHI UHR FE-SEM SU8200 Series) with an accelerating voltage of $3 \mathrm{kV}$ for SEM image capture and $20 \mathrm{kV}$ for EDX mapping. All the Li metal contained electrodes were sealed in a transfer vessel filled with argon before being transferred into the chamber of PHI 5000 VersaProbeIII for XPS analysis. Optically transparent cells for in situ observation were assembled using MPC-Li or bare Li as the cathode during electrodeposition, highly transparent tempered glass as the observation window and paraffin and hot melt adhesive for sealing after injection of electrolyte. Then the optically transparent cells were observed under a digital microscope camera (LEICA ICC50 W). The cross-sectional morphologies in depth were captured with the application of $\mathrm{Ga}^{+}$focused-ion beam at an accelerated voltage of $20 \mathrm{kV}$ and $10 \mathrm{nA}$ current (FIB, FEI Helios 650). All the Li electrodes unloaded from coin cells were washed with a mixed solvent of ethylene carbonate/ dimethyl carbonate (EC:DMC $=1: 1 \mathrm{vol} \%$ ) and thoroughly dried before characterizations. All the characterizations were performed at the room temperature of $25^{\circ} \mathrm{C}$.

Electrochemical measurements. All the electrochemical tests were performed using CR2032 coin cells with a layer of commercial polypropylene (PP) separators $(20 \mu \mathrm{m}$, Celgard 2250). The electrolyte used for electrochemical deposition experiments on MSC-Li and MPC-Li electrodes, optically transparent cells, symmetric cells and full cells was $1 \mathrm{M} \mathrm{LiPF}_{6}$ in ethylene carbonate/dimethyl carbonate (EC:DMC $=1: 1 \mathrm{vol} \%$ ). In deposition experiments tests using perovskite-coated stainless steel foils as electrodes, electrolyte containing $1 \mathrm{M} \mathrm{LiPF}_{6}$ in ethylene carbonate/dimethyl carbonate (EC:DMC $=1: 1 \mathrm{vol} \%$ ) with $2 \mathrm{vol} \%$ fluorinated ethylene carbonate (FEC) was applied. Supplementary Tables 1 and 2 is the data source of the box plots and error bars in Fig. $2 b$. For each row of the data, error bar stands for the upper and lower extremes of the range of values (UE, LE), the box stands for the 75 and 25 percentiles (UQ, LQ) and the square stands for the mean value of each set of data. In Li | LCO cells with $50 \mu \mathrm{m}$ thick Li foil, electrolyte containing $1 \mathrm{M} \mathrm{LiPF}_{6}$ in ethylene carbonate/dimethyl carbonate (EC:DMC = $1: 1 \mathrm{vol} \%$ ) with $1 \mathrm{vol} \%$ fluorinated ethylene carbonate (FEC) was applied. The $\mathrm{Li}_{4} \mathrm{Ti}_{5} \mathrm{O}_{12}$ (LTO) cathodes used for full cell tests were made by blade-coating the $\mathrm{N}$-methyl-2-pyrrolidone (NMP) slurry composed of $80 \mathrm{wt} \% \mathrm{Li}_{4} \mathrm{Ti}_{5} \mathrm{O}_{12}$ powder, $10 \mathrm{wt} \%$ super-P and $10 \mathrm{wt} \%$ poly(vinylidene difluoride) (PVDF) onto carboncoated $\mathrm{Al}$ foil. The areal loading of active material is $3.2 \mathrm{mg} \mathrm{cm}^{-2}$. The $\mathrm{LiCoO}_{2}$ (LCO) cathodes were made by blade-coating the $\mathrm{N}$-methyl-2-pyrrolidone (NMP) slurry composed of $80 \mathrm{wt} \% \mathrm{LiCoO}_{2}$ powder, $10 \mathrm{wt} \%$ super-P and $10 \mathrm{wt} \%$ poly (vinylidene difluoride) (PVDF) onto carbon-coated $\mathrm{Al}$ foil. The areal loading of active material is $20 \mathrm{mg} \mathrm{cm}^{-2}$. Thin $\mathrm{Li}$ foil with thickness of $50 \mu \mathrm{m}$ was purchased from Cellithium company. Electrochemical impedance spectroscopy (EIS) was tested on a VMP-3 (Biologic) electrochemical working station with a frequency range of $100 \mathrm{kHz}$ to $100 \mathrm{mHz}$ and an amplitude of $10 \mathrm{mV}$. Cyclic voltammetry tests were performed on a VMP-3 (Biologic) electrochemical working station with voltage ranges of $1 \mathrm{~V}$ to $-0.1 \mathrm{~V}$ for perovskite-coated stainless steel foils at a scanning rate of $1 \mathrm{mV} \mathrm{s}^{-1}$. Other electrochemical data including cycling tests of $\mathrm{Li} / \mathrm{Li}$ symmetric cells and Li/LTO full cells was collected by a multi-channel cell testing station (CT2001A, Wuhan LAND). All the electrochemical measurements were conducted at a constant temperature of $25^{\circ} \mathrm{C}$.

\section{Data availability}

The data that support the findings of this study are available from the authors on reasonable request, see author contributions for specific data sets.

Received: 13 August 2019; Accepted: 22 March 2020;

Published online: 09 April 2020

\section{References}

1. Armand, M. \& Tarascon, J. M. Building better batteries. Nature 451, 652-657 (2008).

2. Bai, P., Li, J., Brushett, F. R. \& Bazant, M. Z. Transition of lithium growth mechanisms in liquid electrolytes. Energ. Environ. Sci. 9, 3221-3229 (2016).

3. Cheng, X. B., Zhang, R., Zhao, C. Z. \& Zhang, Q. Toward safe Lithium metal anode in rechargeable batteries: a review. Chem. Rev. 117, 10403-10473 (2017).

4. Ding, Y., Cano, Z. P., Yu, A., Lu, J. \& Chen, Z. Automotive Li-ion batteries: current status and future perspectives. Electro Ener. Rev. 2, 1-28 (2019).

5. Lin, D. C., Liu, Y. Y. \& Cui, Y. Reviving the lithium metal anode for highenergy batteries. Nat. Nanotechnol. 12, 194-206 (2017).
6. Lu, J., Chen, Z., Pan, F., Cui, Y. \& Amine, K. High-performance anode materials for rechargeable lithium-ion batteries. Electro Ener. Rev. 1, 35-53 (2018).

7. Liu, J. et al. Pathways for practical high-energy long-cycling lithium metal batteries. Nat. Energy 4, 180-186 (2019).

8. Aurbach, D. Review of selected electrode-solution interactions which determine the performance of $\mathrm{Li}$ and $\mathrm{Li}$ ion batteries. J. Power Sources 89, 206-218 (2000).

9. Peled, E. \& Menkin, S. Review-SEI: past, present and future. J. Electrochem. Soc. 164, A1703-A1719 (2017).

10. Qian, Y. X. et al. Investigations on the electrochemical decomposition of the electrolyte additive vinylene carbonate in $\mathrm{Li}$ metal half cells and lithium ion full cells. J. Power Sources 332, 60-71 (2016).

11. Guo, J., Wen, Z. Y., Wu, M. F., Jin, J. \& Liu, Y. Vinylene carbonate-LiNO3: A hybrid additive in carbonic ester electrolytes for SEI modification on Li metal anode. Electrochem. Commun. 51, 59-63 (2015).

12. Zhang, X. Q., Cheng, X. B., Chen, X., Yan, C. \& Zhang, Q. Fluoroethylene carbonate additives to render uniform $\mathrm{Li}$ deposits in Lithium metal batteries. Adv. Funct. Mater. 27, 165989 (2017).

13. Zheng, G. Y. et al. Interconnected hollow carbon nanospheres for stable lithium metal anodes. Nat. Nanotechnol. 9, 618-623 (2014).

14. Khurana, R., Schaefer, J. L., Archer, L. A. \& Coates, G. W. Suppression of Lithium dendrite growth using cross-linked polyethylene/poly(ethylene oxide) electrolytes: a new approach for practical lithium-metal polymer batteries. $J$. Am. Chem. Soc. 136, 7395-7402 (2014).

15. Tung, S. O., Ho, S., Yang, M., Zhang, R. L. \& Kotov, N. A. A dendritesuppressing composite ion conductor from aramid nanofibres. Nat. Commun. 6, 6152 (2015).

16. Liang, X. et al. A facile surface chemistry route to a stabilized lithium metal anode. Nat. Energy 2, 17119 (2017).

17. Lu, Y. Y., Korf, K., Kambe, Y., Tu, Z. Y. \& Archer, L. A. Ionic-liquidnanoparticle hybrid electrolytes: applications in lithium metal batteries. Angew. Chem. Int. Ed. 53, 488-492 (2014).

18. Lu, Y. Y., Das, S. K., Moganty, S. S. \& Archer, L. A. Ionic liquid-nanoparticle hybrid electrolytes and their application in secondary lithium-metal batteries. Adv. Mater. 24, 4430-4435 (2012).

19. Tu, Z. Y. et al. Fast ion transport at solid-solid interfaces in hybrid battery anodes. Nat. Energy 3, 310-316 (2018).

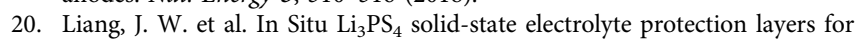
superior long-life and high-rate lithium-metal anodes. Adv. Mater. 30, 1804684 (2018).

21. Bachman, J. C. et al. Inorganic solid-state electrolytes for lithium batteries: mechanisms and properties governing ion conduction. Chem. Rev. 116, 140-162 (2016).

22. Li, X. N. et al. Water-mediated synthesis of a superionic halide solid electrolyte. Angew. Chem. Int. Ed. 58, 16427-16432 (2019).

23. Inaguma, Y. et al. High ionic-conductivity in lithium lanthanum titanate. Solid State Commun. 86, 689-693 (1993).

24. Knauth, P. Inorganic solid Li ion conductors: an overview. Solid State Ion 180, 911-916 (2009).

25. Takada, K. Progress and prospective of solid-state lithium batteries. Acta Mater. 61, 759-770 (2013).

26. Fu, K. et al. Three-dimensional bilayer garnet solid electrolyte based high energy density lithium metal-sulfur batteries. Energ. Environ. Sci. 10, 1568-1575 (2017)

27. Hitz, G. T. et al. High-rate lithium cycling in a scalable trilayer Li-garnetelectrolyte architecture. Mater. Today 22, 50-57 (2019).

28. Cho, H. C. et al. Overcoming the electroluminescence efficiency limitations of perovskite light-emitting diodes. Science 350, 1222-1225 (2015).

29. He, M. et al. Meniscus-assisted solution printing of large-grained perovskite films for high-efficiency solar cells. Nat. Commun. 8, 16045 (2017).

30. Yuan, M. J. et al. Perovskite energy funnels for efficient light-emitting diodes. Nat. Nanotechnol. 11, 872-879 (2016).

31. Zhang, W., Eperon, G. E. \& Snaith, H. J. Metal halide perovskites for energy applications. Nat. Energy 1, 1-8 (2016).

32. Dawson, J. A. et al. Mechanisms of Lithium intercalation and conversion processes in organic-inorganic halide perovskites. ACS Energy Lett. 2, 1818-1824 (2017)

33. Vicente, N. \& Garcia-Belmonte, G. Methylammonium lead bromide perovskite battery anodes reversibly host high Li-ion concentrations. J. Phys. Chem. Lett. 8, 1371-1374 (2017).

34. Yuan, Y. et al. Nature of the band gap of halide perovskites $\mathrm{ABX}_{3}(\mathrm{~A}=$ $\mathrm{CH}_{3} \mathrm{NH}_{3}, \mathrm{Cs} ; \mathrm{B}=\mathrm{Sn}, \mathrm{Pb} ; \mathrm{X}=\mathrm{Cl}, \mathrm{Br}, \mathrm{I}$ ): First-principles calculations. Chin. Phys. B 24, 116302 (2015)

35. Wang, Y. et al. Design principles for solid-state lithium superionic conductors. Nat. Mater. 14, 1026-1032 (2015).

36. Park, C. M., Kim, J. H., Kim, H. \& Sohn, H. J. Li-alloy based anode materials for Li secondary batteries. Chem. Soc. Rev. 39, 3115-3141 (2010). 
37. Shi, J. J., Wang, Z. G. \& Fu, Y. Q. Density functional theory study of diffusion of lithium in Li-Sn alloys. J. Mater. Sci. 51, 3271-3276 (2016).

38. Winter, M. \& Besenhard, J. O. Electrochemical lithiation of tin and tin-based intermetallics and composites. Electrochim. Acta 45, 31-50 (1999).

39. Gong, J. et al. Energy-distinguishable bipolar UV photoelectron injection from LiCl-promoted $\left.\mathrm{FAPbCl}_{3}\right)$ perovskite nanorods. J. Mater. Chem. A 7, 13043-13049 (2019).

40. Fang, Z. S., He, H. P., Gan, L., Li, J. \& Ye, Z. Z. Understanding the role of lithium doping in reducing nonradiative loss in lead halide perovskites. $A d v$. Sci. 5, 1800736 (2018).

\section{Acknowledgements}

We acknowledge the funding support from the National Natural Science Foundation of China (Grants 51571184, 21875236 and 21805268), the Fundamental Research Funds for the Central Universities (Grant WK2060190085), and the joint Funds from Hefei National Synchrotron Radiation Laboratory (Grant KY2060000111). This work was partially carried out at the USTC Center for Micro and Nanoscale Research and Fabrication. G.Z.Z. and Q.W. are grateful to the Supercomputing Center of University of Science and Technology of China for the computing resource. We thank Haitao Liu for his assistance in FIB processing, and Xiu-Xia Wang and Jian Sun for their help with SEM observation.

\section{Author contributions}

H.B.Y. and Y.C.Y. conceived and designed the experiments. G.Z.Z. and Q.W. performed simulations and calculations of Li transport mechanism in perovskite lattices. H.X.J. and Y.C.Y. performed XPS testing and analyzed the data. Y.C.Y., J.T.Y., C.H.J., F.L., H.S.M., and F.Z. carried out the fabrication of perovskite films, solid-state transfer, coin cell assembling and electrochemical tests. K.H.W. and J.S.Y. offered guidance of perovskite syntheses and characterizations. Y.C.Y. designed, assembled and tested the visible batteries and operated characterizations of SEM and EDX mappings. Y.C.Y., G.Z.Z. and Q.W. wrote the original draft. H.B.Y. and Y.C.Y. revised and further wrote the paper. All authors contributed to the data analysis. H.B.Y. directed the project.

\section{Competing interests}

The authors declare no competing interests.

\section{Additional information}

Supplementary information is available for this paper at https://doi.org/10.1038/s41467020-15643-9.

Correspondence and requests for materials should be addressed to G.Z., H.-X.J. or H.-B.Y.

Peer review information Nature Communications thanks the anonymous reviewer(s) for their contribution to the peer review of this work. Peer reviewer reports are available.

Reprints and permission information is available at http://www.nature.com/reprints

Publisher's note Springer Nature remains neutral with regard to jurisdictional claims in published maps and institutional affiliations.

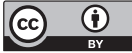

Open Access This article is licensed under a Creative Commons Attribution 4.0 International License, which permits use, sharing, adaptation, distribution and reproduction in any medium or format, as long as you give appropriate credit to the original author(s) and the source, provide a link to the Creative Commons license, and indicate if changes were made. The images or other third party material in this article are included in the article's Creative Commons license, unless indicated otherwise in a credit line to the material. If material is not included in the article's Creative Commons license and your intended use is not permitted by statutory regulation or exceeds the permitted use, you will need to obtain permission directly from the copyright holder. To view a copy of this license, visit http://creativecommons.org/ licenses/by/4.0/.

(C) The Author(s) 2020 